\title{
TOP-N53: A Clinical Drug Candidate for the Treatment of Non-healing Wounds
}

\author{
Reto Naef*, Hermann Tenor, and Guido Koch
}

\begin{abstract}
Chronic non-healing wounds impose a huge burden on patients and health care providers. In spite of improvements in standards of care there are no effective and safe treatments that promote new tissue formation and wound closure in ailments such as diabetic foot ulcer, pressure ulcer, venous leg ulcer or digital ulcer in systemic sclerosis. Endothelial dysfunction, which associates with impaired endogenous nitric oxide formation is assumed to be a main disease mechanism in chronic, non-healing wounds in diabetic and elderly patients as well as in digital ulcers in systemic sclerosis. Topadur Pharma has invented small molecular weight nitric oxidereleasing PDE5 inhibitors, which by modulating a key enzyme system of intracellular signaling may address chronic non-healing wounds. The promising first drug candidate TOP-N53 is currently in early clinical development. Here we describe for the first time the design of TOP-N53 and the synthesis of the clinical GMP batch.
\end{abstract}

Keywords: Diabetic foot ulcer - Digital ulcer · Microcirculation · Nitric oxide · Phosphodiesterase-5 · Pressure ulcer $\cdot$ Regenerative medicine $\cdot$ Soluble guanylate cyclase $\cdot$ TOP-N53 · TOP-52 - Wound healing

\section{Chronic Non-healing Wounds with a High Medical Need}

TOPADUR Pharma AG owns a R\&D platform targeting several aging related diseases, including new therapies for wound healing indications. In spite of current standard of care there remains a high medical need for effective drug treatment of chronic wounds such as diabetic foot ulcer, pressure ulcer, digital ulcers in systemic sclerosis, venous leg ulcers, dental wounds, or scars from surgery performed on elderly patients, where wound healing is delayed or fails.

According to the International Diabetes Federation, about 463 million adults are afflicted with diabetes mellitus; by 2045 this number will rise to 700 million. ${ }^{[1]}$ According to the US CDC report from 2020, the prevalence of type 2 diabetes (T2DM) is $10 \%$ and that of pre-diabetes $33 \%$ in the US population..$^{[2]}$ The WHO reports about 60 million patients with diabetes mellitus in the European Region. Increasing prevalence of diabetes is observed in all ages. Diabetic foot ulcer (DFU) is considered a common and one of the most serious complications of diabetes. ${ }^{[3]}$ Of individuals with diabetes, between $19 \%$ and $34 \%$ are likely to acquire foot ulceration in their lifetime. ${ }^{[4]}$ Every 30 seconds, a lower limb, or part thereof, is lost due to amputation somewhere in the world as a consequence of diabetes..5] Deteriorated cutaneous microcirculation with endothelial dysfunction and impaired angiogenesis has been described in DFU and may contribute to non-healing cutaneous ulcerations and tissue necrosis. ${ }^{[6]}$ Endothelial dysfunction in diabetes is often associated with impaired availability and functionality of endothelial-derived nitric oxide (NO), a physiological gaseous transmitter. ${ }^{[7,8]}$ To improve cutaneous microcirculation and wound angiogenesis, NO may be a therapeutic target in DFU (see Fig. 1).

Systemic sclerosis is a rare, debilitating autoimmune disease of the connective tissue characterized by inflammation, vasculopathy, progressive fibrosis in skin, joints, internal organs with excessive collagen accumulation. The pooled prevalence of systemic sclerosis was reported as 23 cases per 100,000 individuals. ${ }^{[9]}$ Digital ulcers are commonly associated with systemic sclerosis. These are defined as a denuded area of tissue with well-demarcat-

${ }^{\star}$ Correspondence: Dr. R. Naef, E-mail: reto.naef@topadur.com,

Topadur Pharma AG, Grabenstrasse 11A, CH-8952 Schlieren, Switzerland

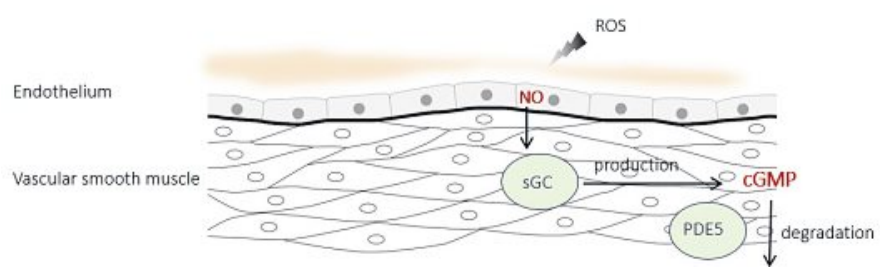

Fig. 1. Endothelial dysfunction and regulation of cGMP. In vascular smooth muscle cells intracellular cGMP is controlled by the balance between synthesis (for example by SGC) and degradation (for example by PDE5). Endothelial dysfunction can be attributed to impaired endogenous NO release from endothelial cells resulting in reduced SGC activation and less cGMP in vascular smooth muscle cells. Finally impaired microcirculation may ensue. Consequently, supply of oxygen and nutrients as well as waste elimination would be compromised that deteriorates tissue regeneration and delays wound healing.

ed borders, involving loss of both the dermis and epidermis often located at the fingertips. These ischemic ulcers are very painful and often result in impaired hand function. About half of those afflicted by systemic sclerosis report a history of digital ulcers and about $10 \%$ have current digital ulcers. ${ }^{[10]}$ Impaired endothelial NO production is considered a characteristic feature of vasculopathy in systemic sclerosis, largely related to dysfunction of systemic sclerosis endothelial cells. ${ }^{[11]}$

For digital ulcers, curative therapies are limited to intravenous iloprost and oral phosphodiesterase-5 (PDE5) inhibitors, while prevention can be achieved with endothelin receptor antagonists. ${ }^{[12]}$ Since reduced NO may cause decreased levels of the cellular second messenger cyclic guanosine-3',5'-monophosphate (cGMP) in endothelial and smooth muscle cells, (oral) phosphodiesterase-5 (PDE5) inhibitors, the soluble guanylate cyclase (sGC) stimulator riociguat (oral), or organic nitrate esters (oral, and topical) have been investigated in clinical trials for their potential therapeutic benefit in secondary Raynaud's phenomenon and digital ulcers in systemic sclerosis ${ }^{[13]}$ (see Fig. 1).

\section{Design of Dual Function NO-releasing PDE5 Inhibitors}

When Topadur Pharma AG was founded in 2015, sGC and PDE5 were validated targets and several drugs were on the mar- 
ket and in development based on the NO/sGC/cGMP signaling cascade. cGMP elevating drugs impart vasodilatation but antifibrotic, anti-inflammatory, anti-proliferative and anti-cancer effects have been described as well. ${ }^{[14]}$

Despite intense R\&D activities by many pharma companies around this signaling cascade it was much to our surprise that examples of PDE5 inhibitor/organic nitrate ester dual function molecules were not known when we started designing NOreleasing PDE5 inhibitors. Our hypothesis was that simultaneous stimulation of NO-sensitive cGMP synthesis combined with inhibition of its degradation would result in an amplified cGMP increase and thus translate into a new quality of cGMP mediated pharmacology. Ultimately, this approach should enable to compensate for the deficiency of endothelial NO in conditions where NO-related endothelial dysfunction occurs exemplified by diabetes mellitus, digital ulcers in systemic sclerosis or aging. Such bifunctional NO-releasing PDE5 inhibitors should be potent and efficacious drug candidates to improve local microcirculation and enable wound healing in indications described above (Fig. 2).

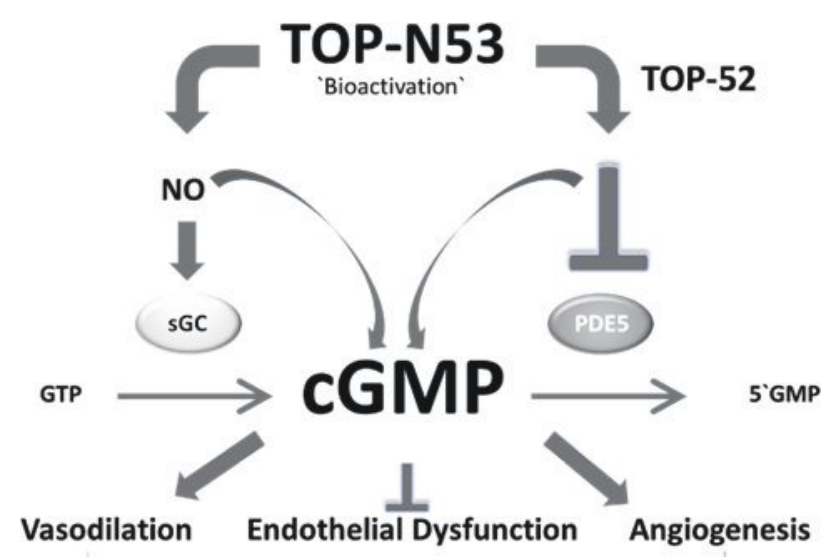

IMPROVED CUTANEOUS MICROCIRCULATION

\section{ACCELERATED WOUND CLOSURE}

Fig. 2. TOP-N53, a highly potent NO releasing PDE5 inhibitor elevates intracellular cGMP by a synergistic interaction between an enhanced cGMP synthesis secondary to stimulation of sGC by the released NO, and reduced cGMP degradation caused by inhibition of PDE5 by TOP-N53 and its active and more potent metabolite, TOP-52 in cells where sGC and PDE5 are expressed. Both NO and TOP-52 are products of the 'bioactivation' of TOP-N53.

The first step towards highly potent PDE5 inhibitors started with structure-guided drug design. There were several published X-ray structures of PDE5/PDE5-inhibitor complexes known. ${ }^{[15,16]}$ The catalytic center of the enzyme contains two divalent metal ions zinc and magnesium. In contrast to the substrate cGMP all the known inhibitors were binding via two hydrate molecules to the active site zinc ion (Fig. 3).

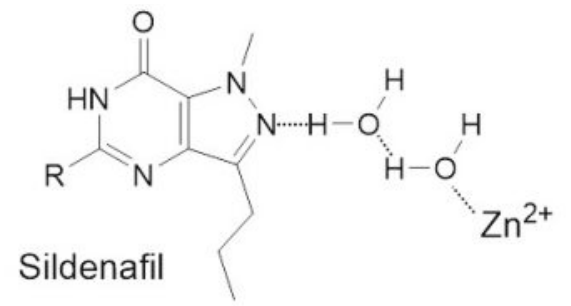

Fig. 3. Binding mode of PDE5 inhibitors to catalytic center in PDE5.
In order to increase the binding affinity of inhibitors on PDE5, we tested the idea to introduce zinc chelating functionalities. The introduction of our newly introduced pyrrolo-triazinone heterocycle to the repertoire of PDE5 inhibitor molecules allowed us to attach the oxime moiety as a zinc chelating functionality (Fig. 4). The study of the inhibitory potency of TOP-N53 as well as computer modeling studies confirmed two possible binding modes (Fig. 5). Removing the water molecules prior to docking resulted in binding where the oxime interacts with the zinc center with a O-Zn distance of $2.98 \AA$ (Fig. 5a), whereas in the alternative binding mode, with the hydrating water layer present in the catalytic site, a different oxime orientation is observed (Fig. 5b) of TOP-N53 with a water mediated interaction to Asp764 and an O-Zn distance of $7.23 \AA$.

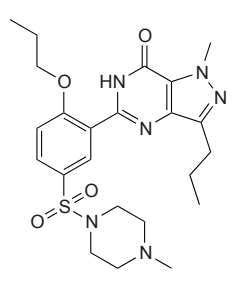

Sildenafil

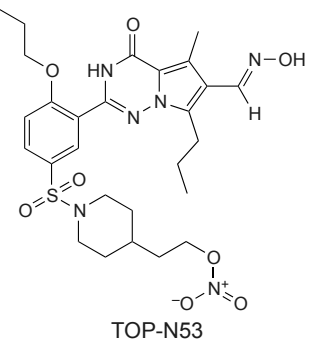

TOP-N53

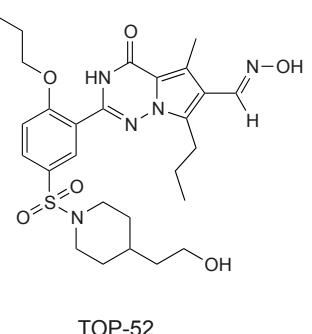

TOP-52
Fig. 4. Molecular structures of Sildenafil, TOP-N53 and TOP-52.

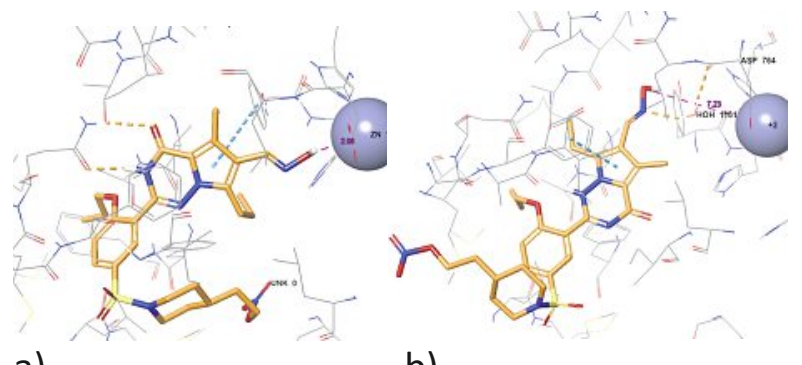

a)

b)

Fig. 5. Docking of TOP-N53 into PDE5. a) without $\mathrm{H}_{2} \mathrm{O}$ solvate b) with $\mathrm{H}_{2} \mathrm{O}$ solvate in the protein.

TOP-52, the active metabolite which is formed together with nitric oxide from TOP-N53 after intracellular bioactivation is a very potent PDE5 inhibitor (Table 1). The increased activity over TOP-N53 can be explained by the additional binding interaction with the polar residues Ser 661 and Gln 663 (Fig. 6). The intracellular release of locally active nitric oxide together with the very

\begin{tabular}{|l|c|}
\hline PDE5 INHIBITOR & IC50 $(\mathbf{n M})$ \\
\hline TOP-N53 & 1.18 \\
\hline TOP-52 & 0.1 \\
\hline VARDENAFIL & 0.33 \\
\hline MIRODENAFIL & 0.56 \\
\hline AVANAFIL & 4.45 \\
\hline TADALAFIL & 6.35 \\
\hline SILDENAFIL & 7.68 \\
\hline
\end{tabular}

Table 1. Inhibition of human recombinant PDE5A1 by TOP-N53, TOP-52 and reference PDE5 inhibitors. IC50 (half-maximum inhibition) values were calculated from concentration-dependent inhibition curves recorded at $0.5 \mu \mathrm{M}$ cGMP and shown as means from at least 3 independent experiments. 


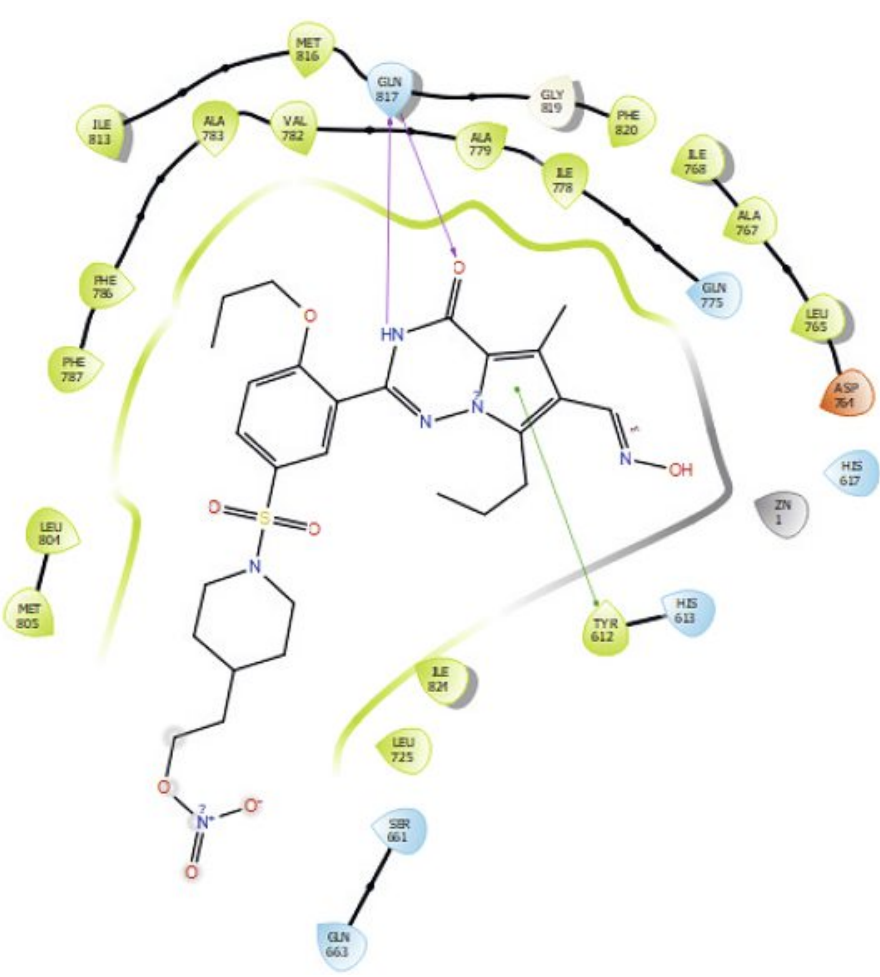

Fig. 6. Binding of TOP-N53 to PDE5.

potent PDE5 inhibitor TOP-52 make Topadur's newly invented enzyme regulation system unique and led to unprecedented efficacy to elevate intracellular cGMP levels.

The GMP synthesis of TOP-N53 (Scheme 1) begins with the formation of amide $\mathbf{5}$ from TBTU-mediated amide formation between benzoic acid $\mathbf{3}$ and aminopyrrole 4. ${ }^{[17]}$ Subsequent basic hydrolysis removes the benzoyl protection and ethyl ester to reveal carboxylic acid 6. Carboxamide $\mathbf{7}$ is produced in a second TBTU mediated amide formation with aqueous ammonia. Cyclisation under basic conditions produces triazine 8. This material undergoes reaction with acetyl nitrate and subsequently hydroxylamine (in a two-step process) to deliver crude TOP-N53 (9). Purification of this material by chromatography controls the overall chemical purity and the unwanted $Z$ oxime isomer to afford TOP-N53. A final recrystallization from ethanol further increases the chemical purity and produces the final TOP-N53 API (10).

TOP-N53 was a potent inhibitor of PDE5 reflected by an IC50 of $1.18 \mathrm{nM}$. TOP-52, its main metabolite after release of NO,
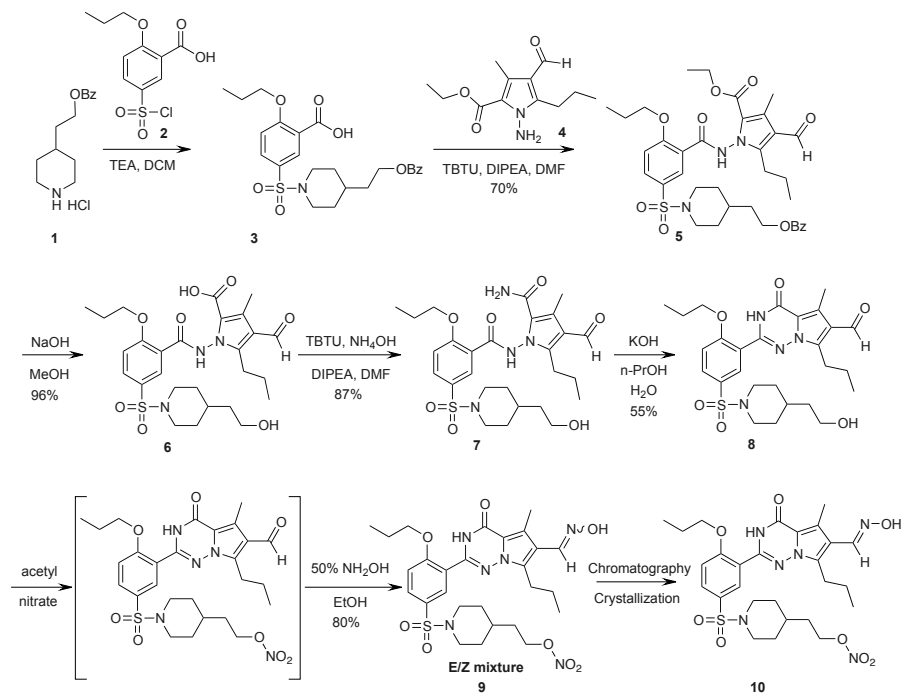

Scheme 1. GMP synthesis of TOP-N53. was revealed to be an even more potent inhibitor of PDE5 with an IC50 of $0.1 \mathrm{nM}$. In fact, compared with a range of marketed PDE5 inhibitors, TOP-52 was the most potent molecule (Table 1).

In cellular assays TOP-N53 was by far more effective than its corresponding PDE5 inhibitor TOP-52 or reference PDE5 inhibitors to increase cGMP as exemplified in washed human platelets (Fig. 7), likely reflecting the synergism inherent in TOP-N53 (Fig. 2).

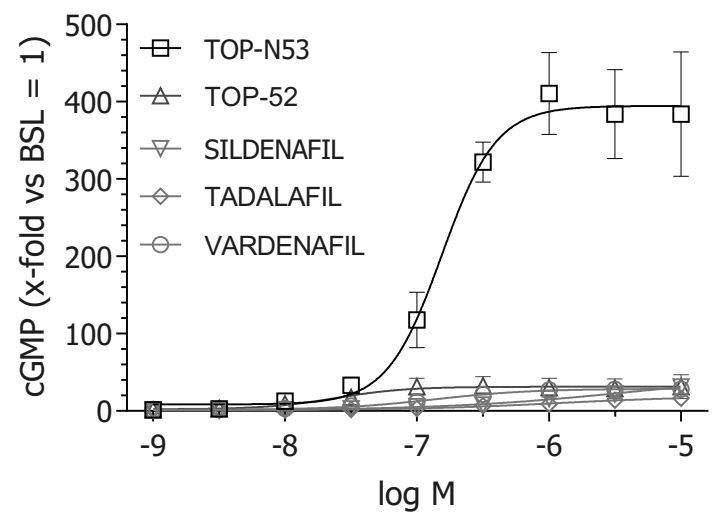

Fig. 7. Effects of TOP-N53, TOP-52 and reference PDE5 inhibitors on cGMP in human platelets. Results are shown as means \pm SEM from platelets of three donors. cGMP was measured after a $2 \mathrm{~h}$ incubation time and in presence of $1 \mu \mathrm{M}$ riociguat and $100 \mathrm{nM}$ BAY 60-7550, a PDE2 inhibitor.

\section{Product/Service and Outlook}

The medicinal chemistry project resulted in a remarkably potent and efficacious drug candidate which translated well into efficacy in preclinical wound healing models. ${ }^{[18]}$ TOP-N53 is currently embarking on clinical development as a topical treatment for wound healing indications.

\section{Topadur}

\section{Pharma AG}

TOPADUR

Topadur Pharma AG, a clinical stage biotech company located in Schlieren, was founded in March 2015 by experienced $\mathrm{R} \& \mathrm{D}$ experts. The unique inventions are small molecular weight drugs, which modulate a key enzyme system of intracellular signal processes. Topadur discovers and develops innovative products to stimulate local blood perfusion for the treatment of wound healing, glaucoma and other ophthalmic indications, as well as oncology to prevent colorectal cancer.

\section{Key Figures}

Founding Year

Location(s)

Legal Form

Founders

$$
2009 \text { (GmbH), } 2015 \text { (AG) }
$$

Headquarter: Schlieren

$\mathrm{AG}$ Christina Attaalla (CFO) Dr. Hermann Tenor (CSO) Dr. Armin Meinzer (former COO) Dr. Armin Hatzelmann
Dr. Reto Naef (CEO \& president) 


\begin{tabular}{|l|l|}
\hline Board of directors & $\begin{array}{l}\text { Pascal Brenneisen } \\
\text { Dr. Dominik Escher } \\
\text { Silvia Scherrer } \\
\text { Dr. Daniel Vasella } \\
\text { Prof. Dr. Günther Wess }\end{array}$ \\
\hline No of employees & 16
\end{tabular}

\section{Key Inventions}

TOP-N53

TOP-N44

TOP-V122

TOP-M119

\section{Awards}

Top100 Swiss Startups

Swiss Tech. Award

Swiss Eco Award

Venture Leaders

China

IPIEC Global

WIPO -

The priority development drug increases local blood flow in the wound area and addresses the impaired blood flow, a central problem in non-healing wounds.

Next milestone: completion of Firstin-Human study

Inhibition of excessive scar formation, targeting a huge medical need after burn wounds.

Next milestone: completion of preclinical development

Ophthalmology:

Corneal wound healing, Glaucoma Oncology: Prevention of colorectal cancer recurrence

Next milestones: completion of preclinical development

Alopecia, skin aging

Next milestone: out-licensing

\section{Contact}

\section{Contact Person}

Address

Email

Website

\section{Acknowledgements}

We gratefully acknowledge the computational chemistry support by Dr. Kiran Boppana (GVK Biosciences, Hyderabad, India).

Received: July 2, 2020

[1] https://www.idf.org/aboutdiabetes/what-is-diabetes/facts-figures.html

[2] https://www.cdc.gov/diabetes/library/features/diabetes-stat-report.html

[3] D. Baltzis, I. Eleftheriadou, A. Veves, Adv. Ther. 2014, 31, 817, https://doi. org/10.1007/s12325-014-0140-X.

[4] D. G. Armstrong, A. J. M. Boulton, S. A. Bus, N. Engl. J. Med. 2017, 376, 2367, https://doi.org/10.1056/NEJMra1615439.

[5] 'IDF Diabetes Atlas', 8th ed. Brussels, International Diabetes Foundation, https://diabetesatlas.org/resources/2017-atlas.html.
[6] M. Roustit, J. Loader, D. Baltzis, W. Zhao, A. Veves, 'Microvascular changes in the diabetic foot', in 'The Diabetic Foot. Contemporary Diabetes', Eds. A. Veves, J. M. Giurini, R. J. Guzman, Springer Nature, 2018, pp 173-188.

[7] A. S. De Vriese, T. J. Verbeuren, J. Van de Voorde, N. H. Lameire, P. M Vanhoutte, Br. J. Pharmacol. 2000, 130, 963, https://doi.org/10.1038/ sj.bjp.0703393.

[8] Y. Shi, P. M. Vanhoutte, J. Diabetes 2017, 9, 434, https://doi. org/10.1111/1753-0407.12521.

[9] L. Zhong, M. Pope, Y. Shen, J. J. Hernandez, L. Wu, Int. J. Rheum. Dis. 2019, 22, 2096, https://doi.org/10.1111/1756-185X.13716.

[10] M. Hughes, A. L. Herrick, Rheumatology (Oxford) 2017, 56, 14, https://doi. org/10.1093/rheumatology/kew047.

[11] Y. Asano, S. Sato, Semin. Immunopathol. 2015, 37, 489, https://doi. org/10.1007/s00281-015-0505-5.

[12] O. Kowal-Bielecka, J. Fransen, J. Avouac, M. Becker, A. Kulak, Y. Allanore, O. Distler, P. Clements, M. Cutolo, L. Czirjak, N. Damjanov, F. del Galdo, C. P. Denton, J. H. W. Distler, I. Foeldvari, K. Figelstone, M. Frerix, D. E. Furst, S. Guiducci, S. Hunzelmann, D. Khanna, M. Matucci-Cerinic, A. L. Herrick, F. van den Hoogen, J. M. van Laar, G. Riemekasten, R. Silver, V. Smith, A. Sulli, I. Tarner, A. Tyndall, J. Welling, F. Wigley, G. Valentini, U. A. Walker, F. Zulian, U. Müller-Ladner, T. Daikeler, E. Lanciano, R. Becvár, M. Tomcik, E. Gińdzieńska-Sieskiewicz, G. Cuomo, M. Iudici, S. Rednic, P. G. Vlachoyiannopoulos, Roberto Caporali, P. E. Carreira, S. Novak, L. Beretta, EUSTAR Coauthors, Ann. Rheum. Dis. 2017, 76, 1327, https:// dx.doi.or/10.1136/annrheumdis-2016-209909.

[13] S. Abraham, V. Steen, Ther. Clin. Risk Manag. 2015, 11, 939, https://doi org/10.2147/TCRM.S82561.

[14] P. Sander, E. M. Becker-Pelster, J. P. Stasch, 'Nitric Oxide', Vol. 77, 1 July 2018, pp. 88-95.

[15] H. Ke, H. Wang, Curr. Top. Med. Chem. 2007, 7, 391, https://doi. org/10.2174/156802607779941242.

[16] H. Wang, Y. Liu, Q. Huai, J. Cai, R. Zoraghi, S. H. Francis, J. D. Corbin, H Robinson, Z. Xin, G. Lin, H. Ke, J. Biol. Chem. 2006, 281, 21469, https:// doi.org/10.1074/jbc.M512527200.

[17] R. Naef, H. Tenor, WO2017085056A1, 2017

[18] M. Greenwald, C. Tacconi, M. Jukic, N. Joshi, P. Hiebert, J. Brinckmann, H. Tenor, R. Naef, S. Werner, J. Invest. Dermatol., 2020, in press, https.//doi org.10.1016/j.jid.2020.05.111.

\section{License and Terms}

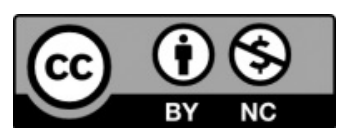

This is an Open Access article under the terms of the Creative Commons Attribution License CC BY_NC 4.0. The material may not be used for commercial purposes.

The license is subject to the CHIMIA terms and conditions: (http:// chimia.ch/component/sppagebuilder/?view = page \&id=12).

The definitive version of this article is the electronic one that can be found at https://doi.org/10.2533/chimia.2020.814 